\title{
Spontaneous Intracranial Hypotension: An Interesting Cause of Intractable Headache
}

\author{
Kiran Kumar*, Himanshi Singh, Sameh Saied, Mohammed Hamdy Ibrahim, \\ Mohammed Khalid, Rifah Anwar Assadi, Sonia Lamichhane, Shaikh Altaf Basha, \\ Rajashree Ganesh, Neha Arora \\ GMC Hospital and Research Centre, Ajman, UAE \\ Email: drkiran k@yahoo.com
}

Received 14 August 2015; accepted 12 September 2015; published 15 September 2015

Copyright (C) 2015 by authors and Scientific Research Publishing Inc.

This work is licensed under the Creative Commons Attribution International License (CC BY). http://creativecommons.org/licenses/by/4.0/

c) (i) Open Access

\begin{abstract}
Intracranial hypotension (ICH) is a benign syndrome which is often under-diagnosed. It is characterized by orthostatic headache which is predominantly occipital. ICH is diagnosed in the presence of a typical history and characteristic imaging findings. Further confirmation by lumbar puncture to document low CSF pressure might be necessary in some cases. Treatment is mainly conservative in the form of bed rest and intravenous saline infusion. However, surgical intervention may be required if conservative measures fail. In this report we presented a case of 42 -year-old male patient who presented with symptoms of orthostatic occipital headache of three-month duration and was subsequently diagnosed with intracranial hypotension based on characteristic MRI findings of pachymeningeal enhancement on gadolinium enhanced MRI of the brain with sagging of the midbrain.
\end{abstract}

\section{Keywords}

Intracranial Hypotension, Headache, CSF Leak, Orthostatic Headache

\section{Introduction}

Intracranial hypotension is a rare condition characterized by orthostatic headache which relieves with supine position. It is due to reduced CSF volume/pressure occurring as result of CSF leakage, either primary/spontaneous or secondary due to lumbar puncture, trauma or surgery. The symptoms are mainly headache which is worse in standing position. Diagnosis is based on characteristic pachymeningeal enhancement on Gadolinium Enhanced MRI of the Brain with sagging of the midbrain. Lumbar puncture might be necessary in certain patients.

"Corresponding author.

How to cite this paper: Kumar, K., Singh, H., Saied, S., Ibrahim, M.H., Khalid, M., Assadi, R.A., Lamichhane, S., Basha, S.A., Ganesh, R. and Arora, N. (2015) Spontaneous Intracranial Hypotension: An Interesting Cause of Intractable Headache. Neuroscience \& Medicine, 6, 130-133. http://dx.doi.org/10.4236/nm.2015.63021 


\section{Case Report}

A 42-year-old Afghanistani male patient, presented to our out-patient department with complaints of occipital headache of three months duration. Headache was severe. It was worse on standing and walking, and was relieved on lying down. There was no history of any fever, nausea or vomiting, visual blurring or any focal neurologic deficits. Patient also denies any nasal discharge or blockage. There was no history of previous surgical procedure, lumbar puncture or trauma. On examination, blood pressure was 130/80 millimeters of mercury, pulse rate was 80 beats per minute, respiratory rate was 16 per minute, axillary temperature was 36.5 degrees centigrade and oxygen saturation was $99 \%$ on room air. Patient was alert and oriented to time, place and person. There were no cranial nerve abnormalities. Motor and sensory system examination was unremarkable. There was no neck stiffness. Romberg sign was negative and gait of the patient was normal. Fundoscopic examination did not show any abnormality. Patient had been on Tab Paracetamol, two tablets four times a day since the past three months. His headache however, remained the same without any sustained improvement.

In view of persistent Orthostatic headache, Gadolinium Enhanced MRI of brain was done which showed sagging of the midbrain, pachymeningeal thickening; both supratentorial and infratentorial, enhancement and engorgement of the dural venous sinuses (Figures 1(a)-(c)).

Based on the clinical features and MRI findings a diagnosis of Intra Cranial Hypotension was made. Patient was managed with bed rest, intravenous hydration and analgesics. Patient had significant symptomatic relief with conservative management. A need for further evaluation with diagnostic lumbar puncture for cerebrospinal fluid pressure testing and possibly a surgical intervention with epidural blood patch (if symptoms persisted) was discussed with the patient. Patient however, did not agree for either lumbar puncture or any surgical intervention and remained on conservative measures. Patient symptoms improved remarkably. Presently patient is symptom free and on regular follow-up.

\section{Discussion}

Intracranial hypotension (ICH) is a generally a benign syndrome and is often under-diagnosed. It is characterized by an orthostatic headache (headache worse at erect posture) and low cerebrospinal volume/pressure. The estimated incidence of spontaneous intracranial hypotension is 5 per 100,000 per year, with a peak age of around 40 years and females are affected thrice as men [1].

Hypo-secretion, hyper-absorption and leakage of CSF are the three different mechanism causing reduction of CSF volume. Recent studies suggest that leakage of CSF is the main cause of ICH. Leakage may result from surgical procedures such as Dural puncture, spinal anesthesia, placement of ventricular peritoneal shunt, trauma or with connective tissue disorder such as Marfan syndrome and Ehlers Danlos Syndrome type 3 [2] [3]. It may also result from various reformer exercises such as Pilates and Yoga when done incorrectly [4]. On the basis of etiology ICH syndrome can be further classified into five categories: 1) primary/spontaneous; 2) post-operative;

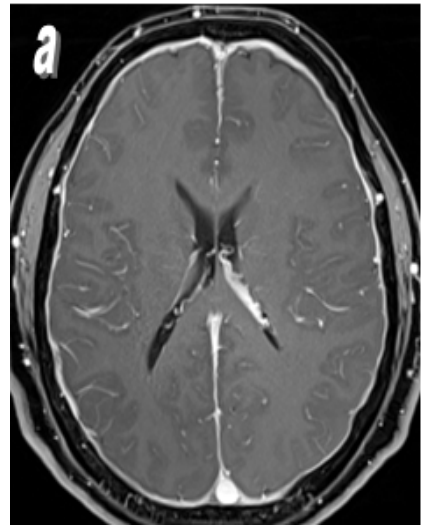

(a)

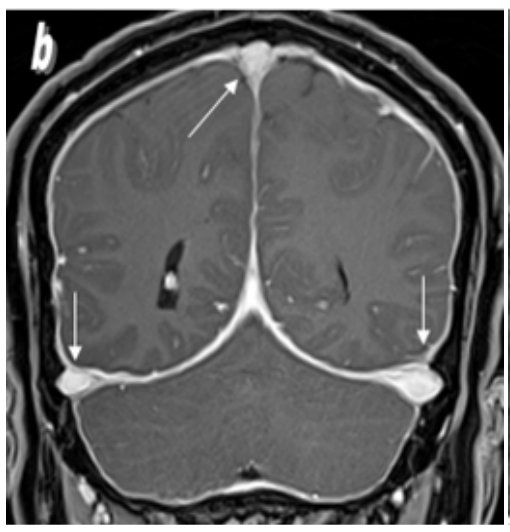

(b)

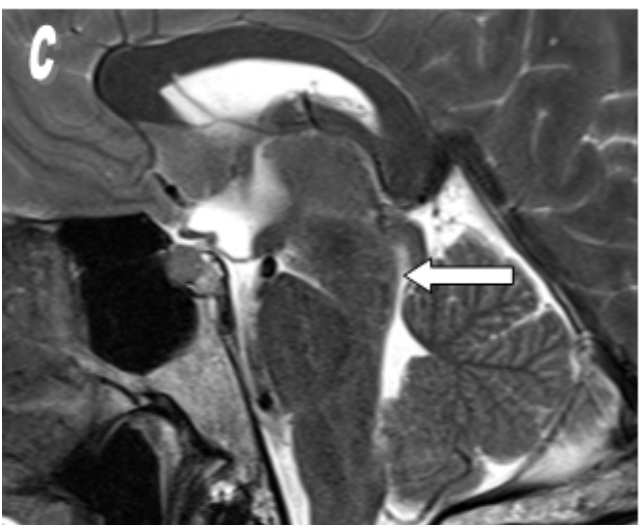

(c)

Figure 1. MRI of the brain before and after IV Gd-DTPA injection. (a) \& (b) Post contrast axial and coronal T1 weighted images with fat suppression showing diffuse pachymeningeal thickening and enhancement and engorgement of the dural venous sinuses (arrows in b). (c) Sagittal T2 weighted image showing sagging of the midbrain (solid Arrow). 
3) post-traumatic; 4) post lumbar puncture and nerve sleeve tear following a fall on buttock; 5) secondary to other systemic condition such as diabetic coma, uremia, dehydration, hyperpnoea, cerebral arteriosclerosis [3] [5]. Spontaneous CSF leakage is uncommon, usually occurring at cervico-thoracic or upper lumbar spine level [6].

All patients with intracranial hypotension present with headache. Although there may be variation in location and nature of headache in patients, majority have headache which is postural. Headache may be at frontal or occipital region or can even be diffuse. Headache is worse at erect position and relieved in supine position [7]. The nature of pain is severe, dull or throbbing and frequently aggravated by upright position, head-shaking, laughing, jugular venous compression, coughing, sneezing and valsalva [8] [9]. Headache is resistant to analgesic. It is often associated with symptoms like vertigo, nausea, vomiting, dizziness, anorexia, neck stiffness, horizontal diplopia, hearing changes, galactorrhea, facial numbness, upper limb radicular symptoms; most of these symptoms are orthostatic in nature [10]. Our patient had the characteristic orthostatic headache which was resistant to analgesic. He however did not have any other associated symptoms such as nausea/vomiting or visual abnormalities.

Diagnosis of ICH is made by lumbar puncture, showing decrease in intracranial pressure often less than 60 $\mathrm{mm}$ of $\mathrm{H}_{2} \mathrm{O}$. In cases of very low ICP, Valsalva maneuver or gentle aspiration with syringe may also be used to withdraw CSF [11]. CSF analysis is often normal but it may show rise in protein concentration, lymphocytic pleocytosis, increased RBC count, which is due to diapedesis of RBC into the CSF due to mechanical disruption and xanthochromia [12].

Magnetic resonance imaging has revolutionized the diagnosis of ICH. The major abnormalities demonstrated on MR imaging studies in patients with ICH are diffuse thickening of the pachymeninges with Gadolinium enhancement, engorgement of venous sinuses, subdural fluid collections, enlargement of the pituitary gland, and downward displacement of the brain [13]. Meningeal enhancement in ICH is thick, linear, without nodularity, and involves the pachymeninges of both the infra- and supratentorial compartments without evidence of involvement of the leptomeninges (no abnormal enhancement around the brainstem, within the sylvian fissures, or in the depth of cerebral sulci) [14]. Our patient had characteristic MRI findings of enhancement of pachymeninges and brainstem descent. Intracranial hypotension if not controlled early, it can lead to cortical vein thrombosis, hyperprolactinemia, subarachnoid hemorrhage, subdural hematoma, cranial nerve palsy [15].

ICH can be managed in most patients with conservative measures. This includes bed rest (Supine Position) to reduce CSF pressure at the site of leakage and therefore allowing healing of the underlying meningeal defects, hydration, analgesic and steroids (Corticosteroids and fludrocortisone) [14]. When conservative treatment fails, invasive procedure such as autologous epidural blood patch (EBP) or surgical closure should be considered. Spontaneous intracranial hypotension can be improved when patients is positioned to 20 degree of trendelenberg [16]. Initial clinical trials have shown that administration of factor XIII promotes the repair of CSF leak sites resulting in improvement of orthostatic headache in patients who were resistant to conservative therapy [17].

Our patient presented with characteristic postural headache which was not relieved with multiple courses of analgesics. Patient had symptomatic relief with conservative treatment; strict bed rest for one week and intravenous followed by oral hydration which was combined with Tab paracetamol/caffeine. Patient did not have any history previous surgery, lumbar puncture or trauma. Therefore, a diagnosis of spontaneous intracranial hypotension was considered.

\section{Conclusion}

Intracranial hypotension is a rare cause of headache. The characteristic orthostatic headache which relives with supine position should raise the suspicion of ICH. Diagnosis can be easily established with typical history and specific MRI findings of pachymeningeal enhancement and brain stem descent. Lumbar puncture, although confirmatory, is not required in all cases and sometimes can aggravate the headache. Conservative measures, such as bed rest and intravenous hydration, are effective in most cases. Patients with persistent CSF leakage or those who do not respond to conservative treatment will be candidates for surgical treatment.

\section{References}

[1] Scheivink, W.I. (2006) Spontaneous Spinal Cerebrospinal Fluid Leaks and Intracranial Hypotension. JAMA, 295, 
2286-2296. http://dx.doi.org/10.1001/jama.295.19.2286

[2] Marcelis, J. and Silberstein, S.D. (1990) Spontaneous Low Cerebrospinal Fluid Pressure Headache. Headache, 30, 192-196. http://dx.doi.org/10.1111/j.1526-4610.1990.hed3004192.x

[3] Greif, S., Mandel, S., Langer, D.J. and Ortiz, R.A. (2014) Spontaneous Intracranial Hypotension Remains an Underdiagnosed Etiology of New-Onset Headache. Practical Neurology, 24-27.

[4] Davis, J., Yanny, I., Chatu, S., Dubois, P., Hayee, B. and Moran, N. (2014) Spontaneous Cerebrospinal Fluid Leak Following a Pilates Class: A Case Report. Journal of Medical Case Reports, 8, 456. http://dx.doi.org/10.1186/1752-1947-8-456

[5] Bruera, O.C., Bonamico, L., Giglio, J.A., et al. (2000) Intracranial Hypotension: The Nonspecific Nature of MRI Findings. Headache, 40, 848-852. http://dx.doi.org/10.1046/j.1526-4610.2000.00154.x

[6] Joen, T.J., Lee, J.D., Lee, B.L., Kim. D.I. and Yoo, H.S. (2001) Radionuclide Cisternography in Spontaneous Intracranial Hypotension with Simultaneous Leaks at the Cervicothoracic and Lumbar Levels. Clinical Nuclear Medicine, 26, 114-116. http://dx.doi.org/10.1097/00003072-200102000-00004

[7] Rahman, M., Bidari, S., Quisling, R. and Friedman, W. (2011) Spontaneous Intracranial Hypotension: Dilemmas in Diagnosis. Neurosurgery, 69, 4-14. http://dx.doi.org/10.1227/NEU.0b013e3182134399

[8] Lay, C., Campbell, K. and Mokri, B. (1997) Low Cerebrospinal Fluid Headache. In: Goadsby, P.J. and Silberstein, S.D., Eds., Headache, Butterworth-Heinemann, Boston.

[9] Raskin, N.H. (1990) Lumbar Puncture Headache: A Review. Headache, 30, 197-200. http://dx.doi.org/10.1111/j.1526-4610.1990.hed3004197.x

[10] Mokri, B. and Posner, J.B. (2000) Spontaneous Intracranial Hypotension: The Broadening Clinical and Imaging Spectrum of CSF Leaks. Neurology, 55, 1771-1772. http://dx.doi.org/10.1212/WNL.55.12.1771

[11] Bell, W.E., Joynt, R.J. and Sahs, A.L. (1960) Low Spinal Fluid Pressure Syndromes. Neurology, 10, 512-521. http://dx.doi.org/10.1212/WNL.10.5.512

[12] Evan, R.W. and Mokri, B. (2002) Spontaneous Intracranial Hypotension Resulting in Coma. Headache, 42, $159-160$. http://dx.doi.org/10.1046/j.1526-4610.2002.02037.x

[13] Mokri, B., Atkinson, J.L., Dodick, D.W., et al. (1999) Absent Pachymeningeal Gadolinium Enhancement on Cranial MRI Despite Symptomatic CSF Leak. Neurology, 53, 402-404. http://dx.doi.org/10.1212/WNL.53.2.402

[14] Rizk, M., El Khatib, M., Yamout, B., Hujeily, E., Ayoub, S., Ayoub, C., et al. (2015) Spontaneous Intracranial Hypotension Syndrome Treated with Fludrocortisone. A \& A Case Reports, 4, 8-11.

[15] Chung, S.J., Kim, J.S. and Lee, M.C. (2000) Syndrome of Cerebral Spinal Fluid Hypovolemia: Clinical and Imaging Features and Outcomes. Neurology, 55, 1321-1327. http://dx.doi.org/10.1212/WNL.55.9.1321

[16] Koch, K.K. and Moran, T.J. (2015) Spontaneous Intracranial Hypotension: Trendelenberg Just May Be the Answer. Military Medicine, 180, e369-e371. http://dx.doi.org/10.7205/MILMED-D-14-00141

[17] Nagatani, K., Takeuchi, S., Wada, K., Mori, K. and Shima, K. (2015) Treatment of Spontaneous Intracranial Hypotension with Intravenous Factor XIII Administration: Initial Clinical Experience. Turkish Neurosurgery, 25, 69-72. 